\title{
Amino Sugars Modify Antagonistic Interactions between Commensal Oral Streptococci and Streptococcus mutans
}

\author{
Lulu Chen,, ${ }^{\text {a,b }}$ Brinta Chakraborty, ${ }^{\mathrm{b}}$ Jing Zou, ${ }^{\mathrm{a}}$ (D) Robert A. Burne, (D) Lin Zeng ${ }^{\mathrm{b}}$ \\ aState Key Laboratory of Oral Diseases, National Clinical Research Center for Oral Diseases, Department of Pediatric Dentistry, West China Hospital of Stomatology, \\ Sichuan University, Chengdu, Sichuan, China \\ ${ }^{b}$ Department of Oral Biology, College of Dentistry, University of Florida, Gainesville, Florida, USA
}

\begin{abstract}
N-Acetylglucosamine (GlcNAc) and glucosamine (GlcN) enhance the competitiveness of the laboratory strain DL1 of Streptococcus gordonii against the caries pathogen Streptococcus mutans. Here, we examine how amino sugars affect the interaction of five low-passage-number clinical isolates of abundant commensal streptococci with S. mutans by utilizing a dual-species biofilm model. Compared to that for glucose, growth on GlcN or GlcNAc significantly reduced the viability of $S$. mutans in cocultures with most commensals, shifting the proportions of species. Consistent with these results, production of $\mathrm{H}_{2} \mathrm{O}_{2}$ was increased in most commensals when growing on amino sugars, and inhibition of S. mutans by Streptococcus cristatus, Streptococcus oralis, or S. gordonii was enhanced by amino sugars on agar plates. All commensals except $S$. oralis had higher arginine deiminase activities when grown on GICN and, in some cases, GlcNAc. In ex vivo biofilms formed using pooled cell-containing saliva (CCS), the proportions of $S$. mutans were drastically diminished when GlcNAc was the primary carbohydrate. Increased production of $\mathrm{H}_{2} \mathrm{O}_{2}$ could account in large part for the inhibitory effects of CCS biofilms. Surprisingly, amino sugars appeared to improve mutacin production by S. mutans on agar plates, suggesting that the commensals have mechanisms to actively subvert antagonism by $S$. mutans in cocultures. Collectively, these findings demonstrate that amino sugars can enhance the beneficial properties of low-passage-number commensal oral streptococci and highlight their potential for moderating the cariogenicity of oral biofilms.
\end{abstract}

IMPORTANCE Dental caries is driven by dysbiosis of oral biofilms in which dominance by acid-producing and acid-tolerant bacteria results in loss of tooth mineral. Our previous work demonstrated the beneficial effects of amino sugars GICNAc and GlcN in promoting the antagonistic properties of a health-associated oral bacterium, Streptococcus gordonii, in competition with the major caries pathogen Streptococcus mutans. Here, we investigated 5 low-passage-number clinical isolates of the most common streptococcal species to establish how amino sugars may influence the ecology and virulence of oral biofilms. Using multiple in vitro models, including a human saliva-derived microcosm biofilm, experiments showed significant enhancement by at least one amino sugar in the ability of most of these bacteria to suppress the caries pathogen. Therefore, our findings demonstrated the mechanism of action by which amino sugars may affect human oral biofilms to promote health.

KEYWORDS amino sugars, bacterial antagonism, clinical commensal isolates, hydrogen peroxide, Streptococcus mutans

$H_{\mathrm{c}}$ undreds of bacterial taxa colonize the surfaces of the oral cavity in threedimensional matrices, termed biofilms (1), the composition of which is driven by adhesive interactions, a variety of synergistic and antagonistic interactions between the microorganisms, and host genetics and behaviors (2). Collectively, these factors deter-
Citation Chen L, Chakraborty B, Zou J, Burne RA, Zeng L. 2019. Amino sugars modify antagonistic interactions between commensal oral streptococci and Streptococcus mutans. Appl Environ Microbiol 85:e00370-19. https:// doi.org/10.1128/AEM.00370-19.

Editor Shuang-Jiang Liu, Chinese Academy of Sciences

Copyright $\odot 2019$ American Society for Microbiology. All Rights Reserved.

Address correspondence to Lin Zeng, Izeng@dental.ufl.edu.

Received 13 February 2019

Accepted 12 March 2019

Accepted manuscript posted online 15

March 2019

Published 2 May 2019 
mine the pathogenic potential of oral biofilms $(3,4)$. Numerous studies now support that the development of the most common oral infectious diseases, dental caries and periodontal diseases, is characterized by a change in the composition and biochemical activities of the microbiota in oral biofilms from one that is rich in health-associated commensals to biofilms that contain substantially increased proportions of opportunistic pathogens or pathobionts. While the factors that drive the formation of periodontopathic biofilms are not fully understood, dental caries results from the establishment of strongly acidogenic (acid-producing) and aciduric (acid-tolerant) biofilms that are selected for by repeated ingestion by the host of fermentable carbohydrates and the resultant recurrent acidification of the biofilms by the organic acids produced during sugar fermentation (5).

Streptococcus mutans is considered a major etiologic agent contributing to the initiation and the progression of dental caries (6). One primary virulence attribute of $S$. mutans is extreme acidification of the environment from the fermentation of an array of carbohydrates $(7,8)$. Another determining factor that enables $S$. mutans to become a successful cariogenic bacterium is its exceptional capacity to form biofilms on teeth, largely facilitated by its robust production of extracellular polymeric substances (EPS) catalyzed by secreted glucosyltransferases (Gtfs) and fructosyltransferase (Ftf) enzymes that generate diffusion-limiting exopolysaccharides (6), and the ability to produce substantial quantities of extracellular DNA (eDNA) (9). The metabolic activities and the matrix combine to create localized low-pH environments that are ideal for S. mutans or other aciduric species to thrive, while these environments suppress the growth of health-associated commensal organisms, which are acid sensitive, unlike cariogenic organisms (2). Furthermore, strains of S. mutans produce multiple lantibiotic and/or non-lantibiotic bacteriocins, collectively referred to as mutacins, which can inhibit the growth of a variety of Gram-positive bacteria (10). While direct evidence from in vivo studies is lacking, it appears that mutacins are critical for allowing S. mutans to establish, persist, and compete with commensal and overtly beneficial bacteria, especially oral streptococci (11). The ComDE two-component system and its cognate signal, competence-stimulating peptide (CSP), comprise the primary quorum-sensing regulatory circuit controlling bacteriocin gene activation, although multiple other factors affect the production of mutacins (12).

As the most abundant species in many dental biofilms, commensal oral streptococci deploy multiple antagonistic strategies against pathogens, creating conditions that are favorable to dental health. For example, in the presence of oxygen, Streptococcus sanguinis, Streptococcus gordonii, and other members of the Mitis group of streptococci produce substantial (millimolar) quantities of hydrogen peroxide $\left(\mathrm{H}_{2} \mathrm{O}_{2}\right)$, which has a potent inhibitory effect on the growth and physiology of S. mutans (4). Likewise, many of the Mitis group streptococci express the arginine deiminase (AD) pathway, which moderates acidification of oral biofilms by releasing ammonia and carbon dioxide while concurrently providing bioenergetic benefits to the producing organisms (13). All $S$. mutans strains lack the AD system. In addition, certain oral streptococci can interfere with intercellular communication systems in a way that reduces the production of mutacins by $S$. mutans and subverts the expression of other key virulence-related phenotypes, including genetic competence. For example, a novel commensal, designated Streptococcus sp. strain A12, isolated from a caries-free human, interferes with the CSP-ComDE signaling system required for mutacin production and the XIP (com $\underline{X}$ inducing peptide) signaling pathway in S. mutans that directly regulates development of genetic competence (14).

Dietary carbohydrates are essential determinants of the cariogenic potential of dental biofilms $(15,16)$. Interestingly, analysis of the microbial composition of the fossil record and ancient calcified dental plaque indicates that dental caries and cariogenic bacteria, respectively, were not common until humans transitioned from a huntergatherer lifestyle to diets richer in natural and refined carbohydrates (17). A western diet, rich in carbohydrates, fuels caries development by greatly increasing the amount and frequency of acid production by oral biofilms. Data are now emerging in support 
of the notion that certain carbohydrates and end products alter biofilm ecology by influencing the antagonistic relationships between health-associated commensals and caries pathogens. For example, glucose, a preferred carbohydrate source, at high concentrations represses $\mathrm{H}_{2} \mathrm{O}_{2}$ production by $\mathrm{S}$. gordonii and reduces its competitiveness against S. mutans (4). S. gordonii significantly reduces $\mathrm{H}_{2} \mathrm{O}_{2}$ production under acidic conditions by diverting carbon flux toward lactic acid production and away from peroxidogenic enzymes, e.g., pyruvate oxidase (18). Similarly, AD expression in many streptococci is sensitive to carbon catabolite repression (CCR) (19). Conversely, certain amino sugars have been shown to enhance the competitiveness of S. gordonii against S. mutans via multiple mechanisms (20).

Amino sugars, including glucosamine (GlcN) and $N$-acetylglucosamine (GlcNAc), are extensively distributed in nature (21), serving as the building blocks for chitin, glycoproteins, bacterial peptidoglycans, and other abundant macromolecules $(22,23)$. Significant quantities of amino sugars and other carbohydrates are also present in human saliva (24-26) and can serve as carbon, nitrogen, and energy sources for many members of the oral microbiota, including oral streptococci. In recent decades, GIcN and GlcNAc have become two of the most widely consumed dietary supplements in the United States and worldwide. Since amino sugars can be rapidly degraded by many oral streptococci and their catabolism leads to release of significant quantities of ammonia, which can raise cytoplasmic and environmental $\mathrm{pH}$, metabolism of these sugars by the oral flora is expected to significantly influence oral biofilm composition and behaviors. Previous work by our group has indicated that metabolism of amino sugars benefits $S$. mutans by improving its acid tolerance (21). However, compared to that of S. mutans, many commensal streptococci have superior abilities to utilize GlcN and especially GIcNAc, which is more common in nature. Also, unlike the case for S. mutans, GIcNAc can elicit catabolite repression in certain commensal oral streptococci as effectively as glucose (27). As a model commensal, S. gordonii DL1 outcompeted S. mutans in both planktonic and biofilm models and was particularly dominant when amino sugars were provided (20). Preliminary genetic analysis also suggested that the cause of the dominance by S. gordonii included more efficient induction of the genes (nag) for the catabolic enzymes and increased expression of the AD pathway (20). However, questions remain as to whether similar influences of amino sugars exist on the other species of abundant commensal streptococci, as well as on complex oral biofilm communities and, if so, what mechanisms and taxa are having the most profound impact on the beneficial or detrimental behaviors of biofilm communities.

Here, we selected a group of bacteria that were recently isolated from supragingival dental plaque of caries-free children and analyzed their interactions with $S$. mutans in the presence and absence of amino sugars. Further, using an ex vivo biofilm model derived from pooled whole saliva, we examined the effects of amino sugars on the persistence and competitive fitness of $S$. mutans in a microcosm community. The results clearly demonstrate that GICN and GICNAc have the potential to modulate the cariogenic potential of dental biofilms, but the effects are complex and highly dependent on the types and proportions of health-associated oral bacteria that are present in the biofilms.

\section{RESULTS}

Utilization of amino sugars for growth by low-passage-number isolates of commensals. Previous studies by our group demonstrated the ability of a variety of oral streptococci, including laboratory reference strains, to utilize amino sugars for growth (20). To achieve a more comprehensive understanding of metabolism of amino sugars by dental biofilms, we obtained five clinical isolates derived from the supragingival plaque of caries-free subjects, each representing an abundant species of oral streptococci based on their 16S rRNA sequence: Streptococcus sanguinis BCC04, S. gordonii BCC09, Streptococcus cristatus BCC13, Streptococcus oralis BCC02, and Streptococcus intermedius BCC01. Phylogenomic analysis of the genome sequences of these strains has confirmed the assignment of species (28). As part of an ongoing study 
TABLE 1 Strains and plasmids used in this study

\begin{tabular}{lll}
\hline Strain or plasmid & Relevant characteristic(s) & Source or reference \\
\hline $\begin{array}{l}\text { Strains } \\
\text { Commensals }\end{array}$ & & \\
S. sanguinis BCC04 & $\mathrm{AD}(++)$; antagonism (+) & Clinical strain \\
S. gordonii BCC09 & $\mathrm{AD}(+++)$; antagonism (++) & Clinical strain \\
S. cristatus BCC13 & $\mathrm{AD}(++)$; antagonism $(+++)$ & Clinical strain \\
S. oralis BCC02 & $\mathrm{AD}(+)$; antagonism (+) & Clinical strain \\
S. intermedius BCC01 & $\mathrm{AD}(+++)$; antagonism (-) & Clinical strain \\
S. sanguinis SK150 & Wild-type reference strain & Laboratory stock \\
S. mutans & Wild-type reference strain & ATCC \\
UA159 & gtfA::Km & This study \\
UA159-Km & &
\end{tabular}

Plasmid

PBGK2 Streptococcus integration vector, $\mathrm{Km}^{\mathrm{r}} \quad 56$

${ }^{a}$ Five clinical strains (BBC01 to $\mathrm{BBC13}$ ) were isolated from supragingival dental plaque of caries-free subjects. $\mathrm{AD}$ activity and antagonism against $\mathrm{S}$. mutans UA159 in $\mathrm{BHI}$ medium was assessed and recorded with the following symbols: - , none; + , low; ++ , moderate; +++ , high.

dissecting probiotic mechanisms of beneficial oral bacteria, these strains were assessed for traits that have been associated with dental health, including expression of the arginine deiminase pathway and antagonism of growth of $S$. mutans (Table 1).

First, to assess their capacity to utilize amino sugars, the five isolates were cultured to exponential phase in brain heart infusion $(\mathrm{BHI})$ broth and then diluted into the chemically defined medium FMC (21) constituted with $20 \mathrm{mM}$ glucose (Glc), GlcN, or GlcNAc. As shown in Fig. 1, S. sanguinis BCC04, S. gordonii BCC09, and S. cristatus BCC13 grew similarly well in all three carbohydrates, with only modestly faster growth on Glc than on GlcNAc. By comparison, S. oralis BCC02 and especially S. intermedius BCC01 displayed significantly lower growth rates on GICNAC, along with slightly longer lag phases and lower yields, than on Glc or GlcN. Nevertheless, compared with the growth phenotype of S. mutans on GICNAc (20), the commensal isolates showed no apparent defect in catabolizing amino sugars under these test conditions.

Amino sugars influence competition between S. mutans and commensals in biofilms. To investigate whether amino sugars can influence the interactions between the selected commensals and S. mutans, a dual-species biofilm model (20) was established. Bacterial cultures in exponential phase were prepared in $\mathrm{BHI}$, and each commensal isolate, alone or in a 1:1 ratio with S. mutans UA159-Km (29), was used to inoculate biofilm medium (BM) supplemented with $18 \mathrm{mM}$ Glc and $2 \mathrm{mM}$ sucrose (BMGS) to form biofilms on glass surfaces.

S. sanguinis BCCO4 formed mature biofilms with extensive structures, whereas the four other commensals produced only sparse microcolonies under these conditions, regardless of the primary carbohydrate source (see Fig. S1 in the supplemental material). In contrast, S. mutans UA159 alone formed relatively thick biofilms, albeit consisting of significant proportions of dead cells (Fig. 2A). Further, S. mutans formed comparable amounts of biofilms on three different sugars, as indicated by both microscopy and the results of CFU enumeration (Table S1). We posit that the four commensals that did not form robust biofilms could not do so, at least in part because they lack the ability to efficiently convert sucrose into water-insoluble glucans, which are known to enhance adherence to glass and biofilm maturation.

Even though different biofilm phenotypes were detected when S. mutans was combined with each of these commensals, some similar patterns were uncovered. Nearly all dual-species biofilms displayed the most extensive structures with Glc as the primary carbohydrate source, especially biofilms composed of S. intermedius BCC01 or S. cristatus BCC13 and S. mutans (Fig. 2A). In contrast, notably thinner biofilms were observed when the dual-species biofilms were formed using media formulated with GlcN or GlcNAc.

Consistent with their overall appearance, total numbers of CFU in these biofilms were strongly influenced by the carbohydrate source. Significantly lower numbers of 


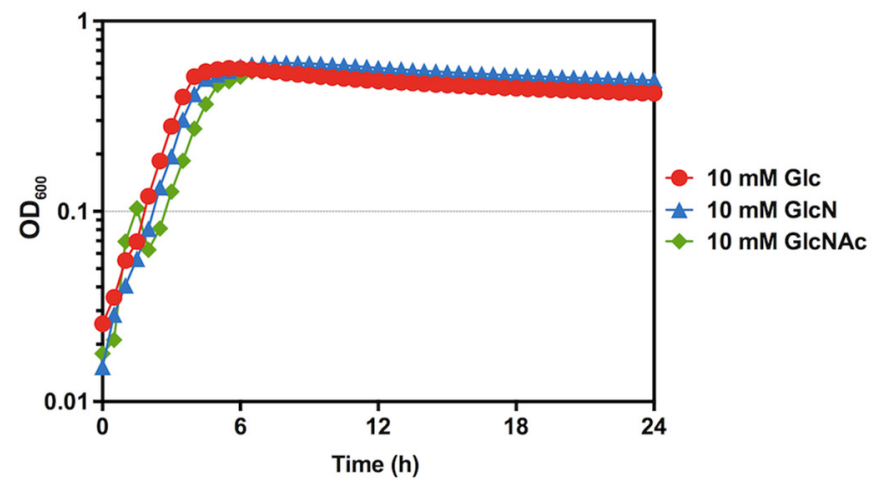

S. sanguinis BCC04
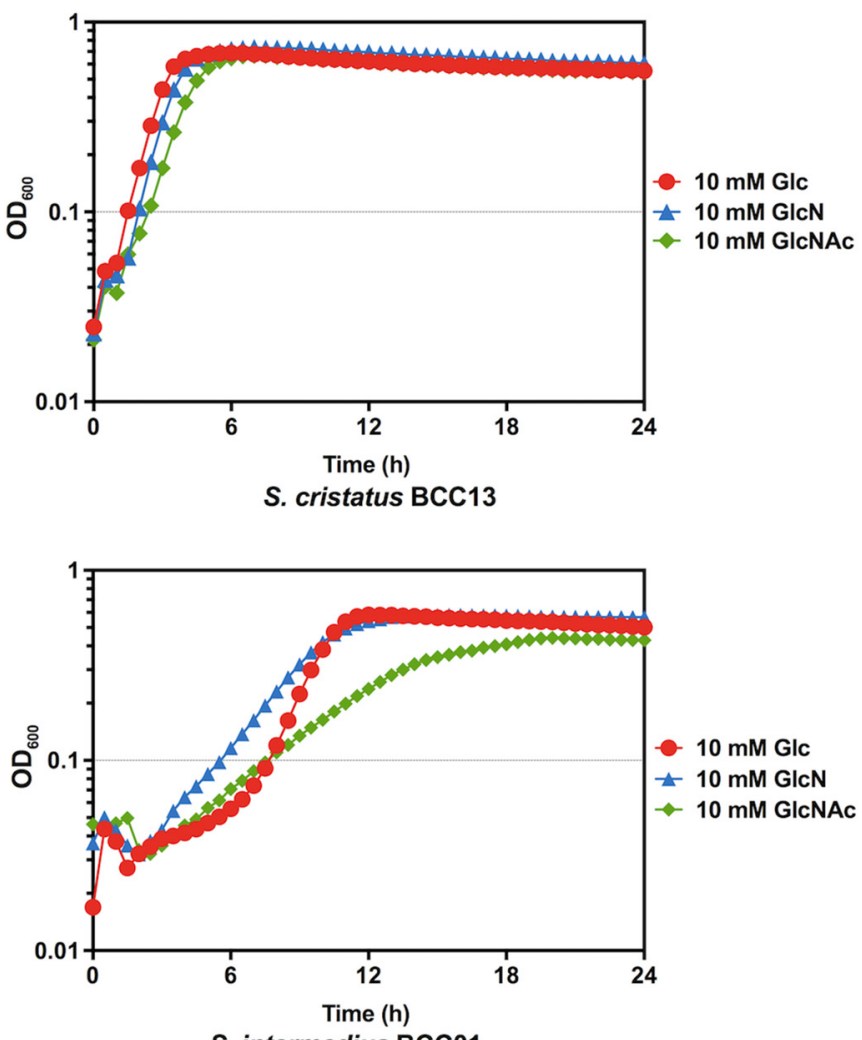

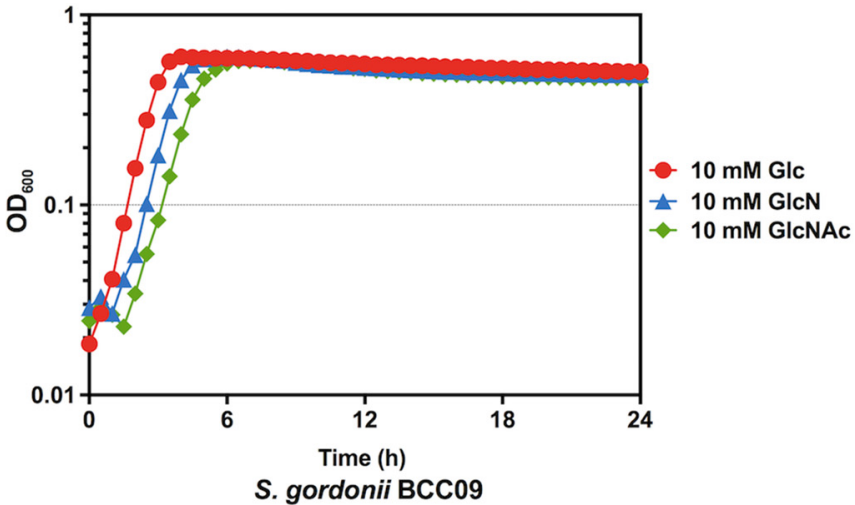

S. gordonii BCC09

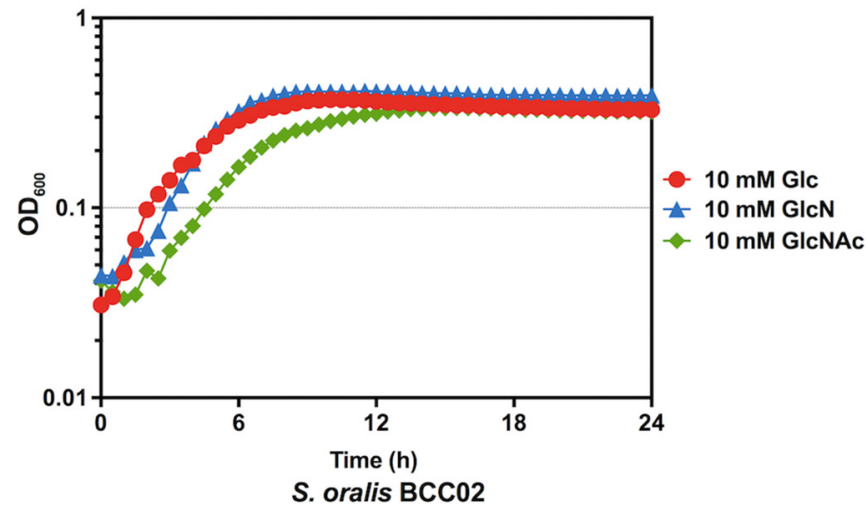

FIG 1 Growth curves. Commensal strains were cultured in $\mathrm{BHI}$ until the $\mathrm{OD}_{600}$ reached 0.5. Cultures were then diluted 1:50 into FMC medium supplemented with $10 \mathrm{mM}$ Glc (red), GlcN (blue), or GlcNAc (green). The $\mathrm{OD}_{600}$ was monitored using a Bioscreen $\mathrm{C}$ with readings taken every 30 min.

CFU were obtained in dual-species biofilms composed of S. cristatus BCC13, S. oralis BCC02, or S. intermedius BCC01, together with S. mutans, when amino sugars were the supporting carbohydrate source. Cocultures of S. mutans with S. sanguinis BCCO4 produced significantly fewer total CFU on GIcN than Glc; however, the results with Glc and GlcNAc were similar for BCCO4.

The antibiotic marker engineered into strain UA159-Km allowed us to differentiate the two populations of bacterial cells in each system. In nearly all cases, when contrasted with Glc, one or both amino sugars yielded lower CFU of UA159-Km in the biofilms, with significant reductions in the proportions of total CFU comprised of UA159-Km, except for S. intermedius (Fig. 2B). For example, amino sugars allowed S. gordonii BCC09 to become the dominant species in the dual-species biofilms $(80 \%$ or higher), whereas the opposite was true with Glc. As an exception, cocultures of $S$. mutans with S. sanguinis BCCO4 showed no significant change in S. mutans cell counts on GlcNAc relative to those on Glc. 
A

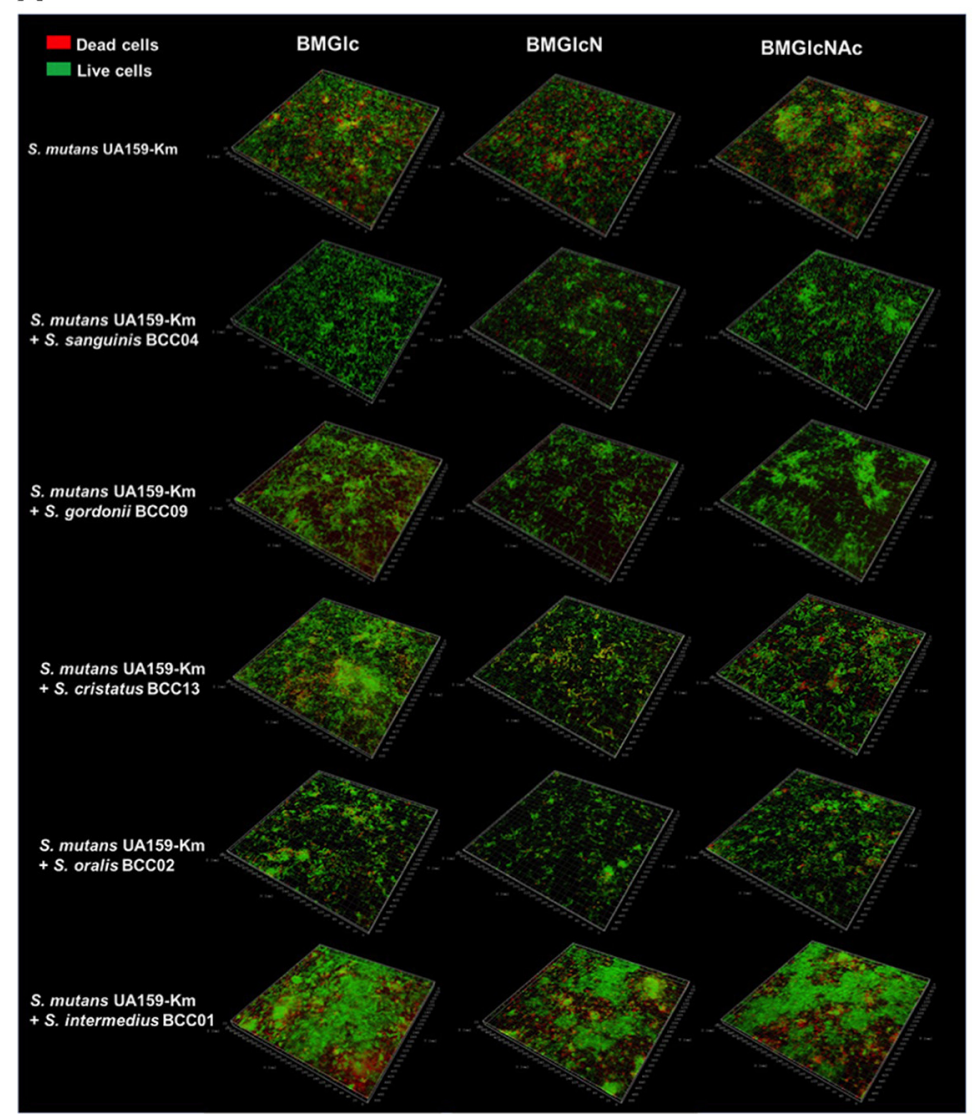

B


FIG 2 Dual-species biofilms formed using clinical commensals and S. mutans. Each commensal was mixed with UA159-Km in equal proportions and inoculated into BM supplemented with $18 \mathrm{mM}$ Glc and $2 \mathrm{mM}$ sucrose (BMGS) to form biofilms overnight on glass coverslips. BMGS was then replaced by BM supplemented with $20 \mathrm{mM} \mathrm{Glc}$, GlcN, or GICNAc and incubated for another $24 \mathrm{~h}$ before visualization by confocal laser scanning microscopy (CLSM) after LIVE/DEAD staining (A) and CFU quantification of both species (B). Biofilms formed by UA159-Km alone were included for comparison. Values show the percentages of the populations constituted by commensal bacteria (top) and S. mutans (bottom), respectively. Asterisks indicate statistically significant differences in the viable counts of S. mutans compared to that on BMGlc. ${ }^{*}, P<0.05 ;{ }^{* *}, P<0.01 ;{ }^{* * *}, P<0.001 ;{ }^{* * * *}, P<0.0001$.

Growth on amino sugars increases $\mathrm{H}_{2} \mathrm{O}_{2}$ production by peroxidogenic commensals. It is believed that the $\mathrm{H}_{2} \mathrm{O}_{2}$ produced by commensal streptococci is a potent inhibitor of S. mutans and other oral pathobionts in vivo $(30,31)$. To begin dissecting the mechanisms by which amino sugars could improve the survival of commensals in cocultures with $\mathrm{S}$. mutans, the amount of $\mathrm{H}_{2} \mathrm{O}_{2}$ produced in liquid cultures by each commensal was quantified (Fig. 3).

S. gordonii BCC09 produced significantly more $\mathrm{H}_{2} \mathrm{O}_{2}$ when growing in TY medium supplemented with amino sugars, especially GlcNAc, than cells growing on glucose $(P<0.01)$. This finding is consistent with a previous report of a laboratory strain of $S$. gordonii (DL1) during its interactions with S. mutans (20). For S. cristatus BCC13 and S. oralis $\mathrm{BCC} 2$, relatively high concentrations of $\mathrm{H}_{2} \mathrm{O}_{2}$ were generated under all culture conditions, a result that is consistent with their outstanding ability to inhibit $S$. mutans in the biofilm model (Fig. 2). Importantly, more $\mathrm{H}_{2} \mathrm{O}_{2}$ was generated by $\mathrm{BCC} 13$ and $\mathrm{BCCO} 2$ in the presence of either amino sugar, although some of these differences were not statistically significant. S. intermedius $\mathrm{BCC} 01$, on the other hand, was unable to produce detectable levels of $\mathrm{H}_{2} \mathrm{O}_{2}$ (data not shown), consistent with what has been described for some other $\mathrm{S}$. intermedius strains (32). Interestingly, S. sanguinis strain $\mathrm{BCC} 4$ also produced more $\mathrm{H}_{2} \mathrm{O}_{2}$ when growing on either amino sugar, although it was only GlcN that enhanced its competitiveness in the biofilm model (Fig. 2B).

Competition between S. mutans and clinical commensals on agar. Environmental factors such as oxygen tension and carbohydrate availability are known to affect not 


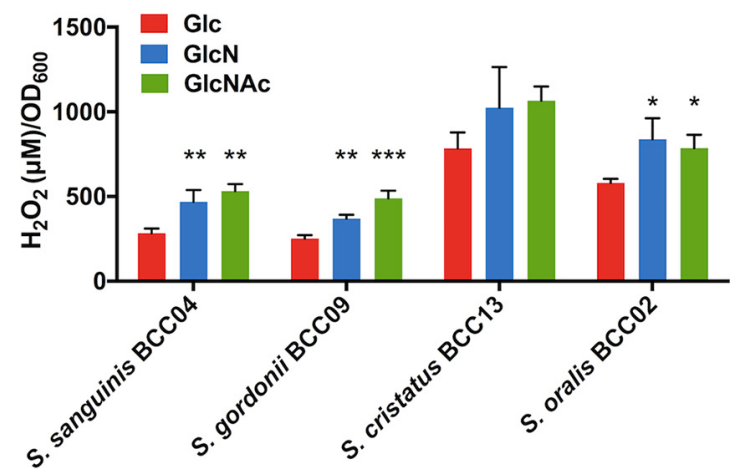

FIG $3 \mathrm{H}_{2} \mathrm{O}_{2}$ production. Cells were cultivated to early exponential phase in TY supplemented with $20 \mathrm{mM}$ $\mathrm{Glc}$, GlcN, or GlcNAc and exposed to vigorous aeration, and the concentrations of $\mathrm{H}_{2} \mathrm{O}_{2}$ in the supernates were determined as described in Materials and Methods. Asterisks indicate statistically significant differences in $\mathrm{H}_{2} \mathrm{O}_{2}$ levels relative to those on glucose. ${ }^{*}, P<0.05 ;{ }^{* *}, P<0.01$; ${ }^{* * *}, P<0.001 ;{ }^{* * *}, P<$ 0.0001 .

only the production of $\mathrm{H}_{2} \mathrm{O}_{2}$ by peroxidogenic species, such as $\mathrm{S}$. gordonii (33), but also the production of mutacins (bacteriocins) by S. mutans (34). We performed a series of pairwise competition assays to assess the effects of carbohydrate source and availability of oxygen on competition between the commensals and S. mutans. S. mutans UA159 and commensals were inoculated onto TY agar plates supplemented with $20 \mathrm{mM} \mathrm{Glc}$, GlcN, or GlcNAc in three different ways: (i) S. mutans was spotted and incubated for $24 \mathrm{~h}$ before the commensal was spotted nearby, and plates were incubated an additional $24 \mathrm{~h}$; (ii) vice versa, i.e., the commensal was spotted first; and (iii) both species were spotted simultaneously and incubated for $24 \mathrm{~h}$.

As shown in Fig. 4, in an aerobic environment, S. mutans generally inhibited the growth of commensal bacteria when it was spotted first, irrespective of the carbohydrate source. However, when commensals were inoculated first, differential effects of the carbohydrate source on competition of commensals with S. mutans were evident. For example, S. cristatus $\mathrm{BCC} 13$ and S. oralis BCC02 showed significantly stronger inhibition of S. mutans on plates containing GlcN than Glc. For S. gordonii, the enhanced antagonism of $S$. mutans was particularly significant on GlcNAc-containing plates. In contrast, S. sanguinis BCCO4 showed a slight, positive response to GlcNAc, and S. intermedius BCC01 showed no inhibition of S. mutans, regardless of the carbohydrate source. Finally, when the commensals and $S$. mutans were spotted the same time, $S$. gordonii, S. cristatus, and S. oralis showed reduced evidence of antagonism, and only a modest effect of carbohydrate source was observed. Together, these results suggested that at least one amino sugar favored three commensals in antagonizing S. mutans on agar plates if the commensals were allowed to become established prior to plating of S. mutans.

To assess to what degree the presence of oxygen, and therefore the ability of the commensals to produce $\mathrm{H}_{2} \mathrm{O}_{2}$, contributed to these antagonistic activities, the same assays were conducted under anaerobic conditions. The results (Fig. 4) showed that most clinical commensals lost their ability to inhibit S. mutans regardless of the sequence of inoculation or carbohydrate source. Notably, when S. mutans was spotted first, it showed improved inhibition of all commensals when amino sugars were used in the plates compared to that of plates with glucose as the primary carbohydrate source. Thus, under more anaerobic conditions and/or in a reduced redox environment, the presence of amino sugars could favor competition of $S$. mutans against beneficial commensals.

AD activities by commensal isolates. In S. gordonii DL1, AD expression is repressed by glucose (19) but not by growth on amino sugars (20). To assess the effect of amino sugars on $A D$ expression by the commensals in this study, the isolates were grown in TY medium containing $20 \mathrm{mM} \mathrm{Glc}$, GlcN, GlcNAc, or galactose, each supplemented with 


\begin{tabular}{ccc}
\multicolumn{3}{c}{ Aerobic } \\
\hline TY- & TY- & TY- \\
Glc & GIcN & GIcNAc
\end{tabular}

\begin{tabular}{ccc}
\multicolumn{3}{c}{ Anaerobic } \\
\hline TY- & TY- & TY- \\
Glc & GIcN & GIcNAc
\end{tabular}

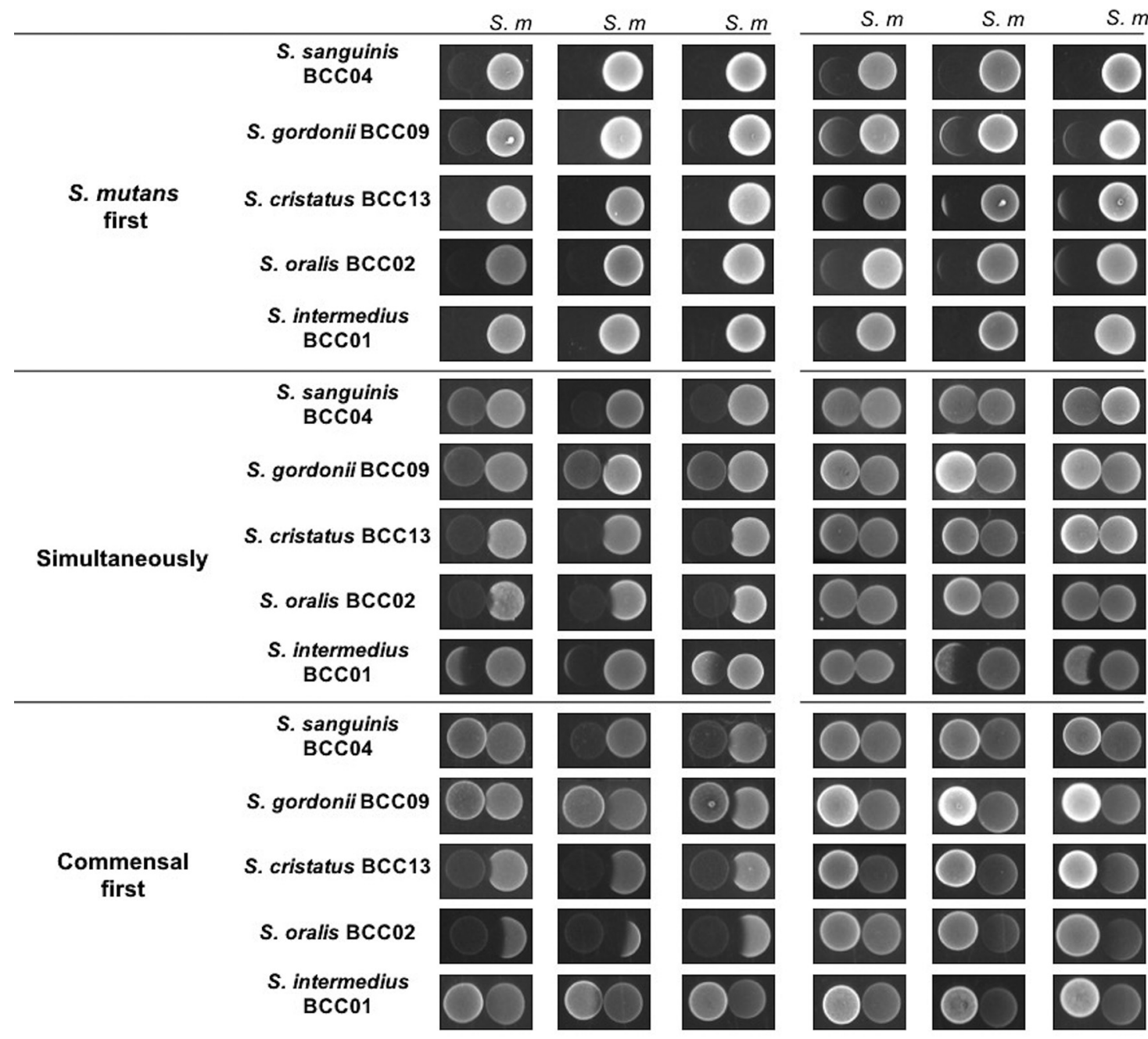

FIG 4 Competition assays between S. mutans and clinical commensals. Commensals and S. mutans UA159 were spotted on TY agar plates containing $20 \mathrm{mM} \mathrm{Glc}$, GlcN, or GlcNAc and incubated under aerobic or anaerobic conditions. The competitions were initiated with colonization by UA159 (S. mutants first) or by commensals (commensal first) alone for $24 \mathrm{~h}$ and then followed by the other or by both (simultaneously).

$10 \mathrm{mM}$ arginine. The $\mathrm{pH}$ of the supernatant fluid was recorded after the cultures were harvested in exponential phase (Fig. S2). Galactose was used as a positive control, as it does not significantly repress AD expression (35). For S. sanguinis BCC04, S. cristatus BCC13, and most notably S. gordonii BCC09 and S. intermedius BCC01, addition of GlcN resulted in enhanced $A D$ activity compared to that of cells growing on Glc (Fig. 5). AD activity was modestly higher on GlcNAc, although the differences were not statistically significant, for S. gordonii BCC09. Notably, the pattern of culture $\mathrm{pH}$ values for each strain corresponded with AD activity. Specifically, galactose-grown cultures presented the highest final $\mathrm{pH}$ values of all the sugars, and for S. gordonii, S. cristatus, and S. intermedius, use of amino sugars also resulted in elevated $\mathrm{pH}$ compared to that of glucose-grown cultures (Fig. S2). The lack of efficient induction of AD in the presence of GICNAc relative to GIcN has been observed for S. gordonii DL1 (20); however, the molecular basis for this difference is not defined. S. oralis BCC02 produced negligible amounts of AD activity under all conditions, and its overall $\mathrm{pH}$ values were significantly lower than those of the other strains. 


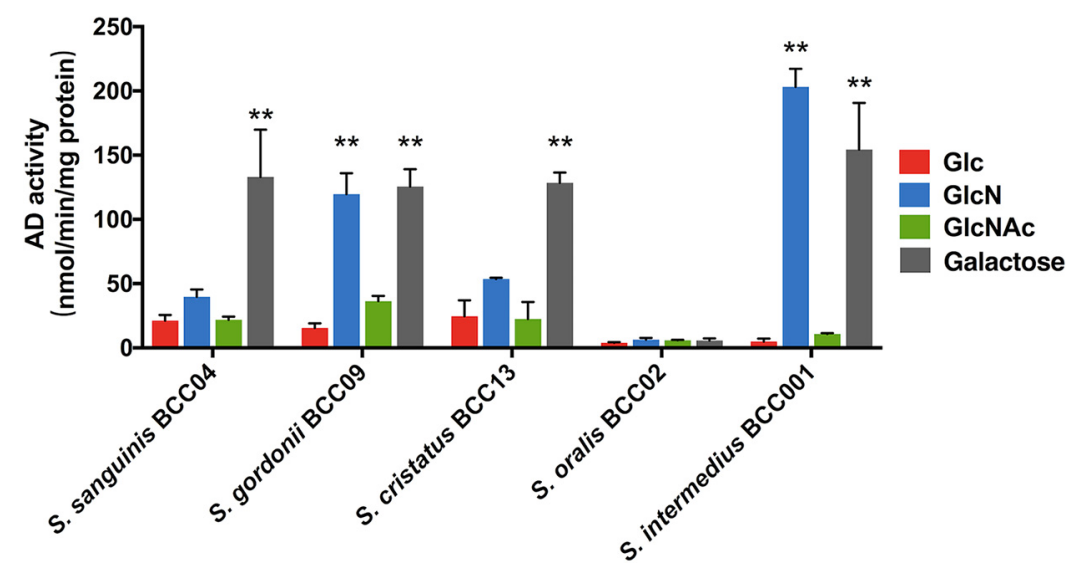

FIG 5 Arginine deiminase (AD) activity. For AD assays, commensals were grown to exponential phase in liquid TY supplemented with $20 \mathrm{mM} \mathrm{Glc}$, GIcN, GlcNAc, or galactose, in addition to $10 \mathrm{mM}$ arginine. Asterisks denote statistically significant difference compared to cultures prepared with glucose. ${ }^{*}, P<$ $0.05 ;{ }^{* *}, P<0.01 ;{ }^{* * *}, P<0.001 ;{ }^{* * *}, P<0.0001$.

Bacteriocin production by S. mutans is affected by amino sugars. S. mutans secretes various antimicrobial peptides, called mutacins, that are capable of inhibiting the persistence of certain commensal streptococci $(11,36)$, an activity that can be countered by some commensals $(14,37)$. In order to investigate if production of mutacins is affected by amino sugars or the presence of the five organisms tested here, a soft-agar overlay assay was performed using S. sanguinis SK150 as the indicator strain (14). S. mutans UA159 alone, or UA159 together with one commensal, was inoculated on agar plates containing Glc, GlcN, or GlcNAc. After 1 day of incubation, soft agar containing S. sanguinis SK150 cells was overlaid and the incubation was continued for $24 \mathrm{~h}$ before measuring zones of inhibition. The soft agar used to prepare SK150 suspensions was formulated to contain the same carbohydrate as that in the agar plates, and SK150 grew equally well on all three sugars (Fig. S3). As shown in Fig. 6, when S. mutans was used alone, it produced generally larger inhibition zones under aerobic conditions versus anaerobic incubation when tested on the same carbohydrates. Interestingly, the largest zones of inhibition occurred on GIcN plates, followed by those on GlcNAc, which were slightly larger than those on Glc plates. These results were consistent with our previous study and may be associated with the effects of preferred carbohydrates, $\mathrm{pH}$, and oxygen on mutacin gene expression and activity (21).

Coinoculation with commensals under aerobic conditions largely abolished the zone of inhibition of SK150 by S. mutans in all cases except for S. intermedius, regardless of the carbohydrate sources (Fig. 6). Conversely, incubation of the plates under anaerobic conditions significantly reduced, but did not completely remove, the effects of the four commensals on the reduction of the size of the zones of inhibition. These results were consistent with $\mathrm{H}_{2} \mathrm{O}_{2}$ being a dominant factor in the ability of the commensals to inhibit S. mutans. Different from the results of earlier assays (Fig. 2 and 4), among the four commensals that influenced the size of the zones of inhibition, similar levels of efficiency were observed under aerobic conditions, regardless of the carbohydrate species. However, under anaerobic conditions, these four effective strains showed the greatest impact on reducing the zone of inhibition when assayed on plates supplied with GlcN, followed by GlcNAc. Collectively, these results suggest that while $\mathrm{H}_{2} \mathrm{O}_{2}$ remains an effective mechanism for commensals to reduce the antagonistic capacity of S. mutans, additional factors are produced by particular commensals that are more dependent on carbohydrate source to affect production or effectiveness of mutacins by S. mutans.

GIcNAc adversely affects persistence of S. mutans in an ex vivo biofilm model. S. mutans persists in a dynamic biofilm environment with hundreds of bacterial taxa, most of which have not been well characterized (38-40). To better mimic the complex 


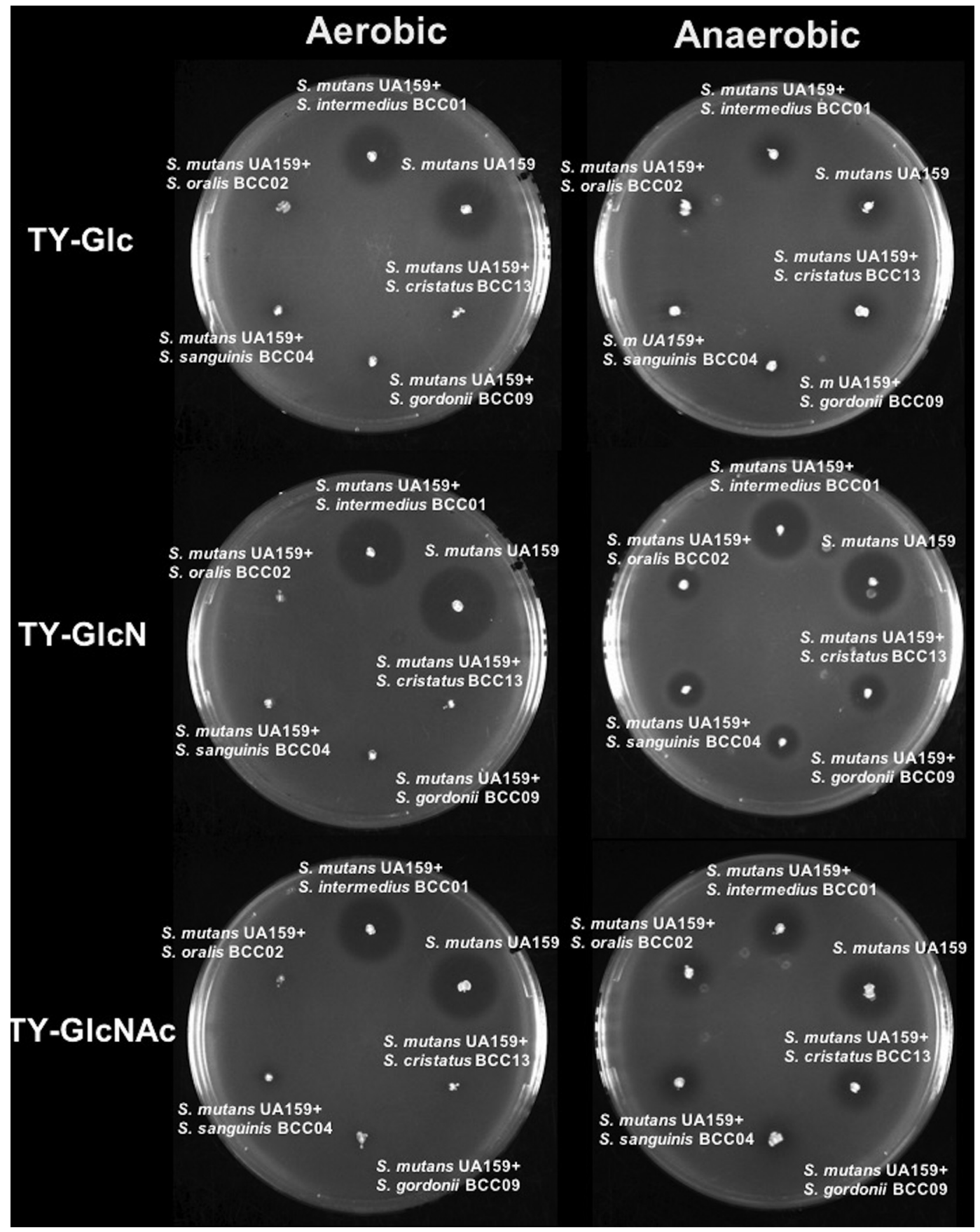

FIG 6 Plate-based bacteriocin assay. Washed cells of each clinical commensal were mixed with UA159 at a 1:1 ratio based on optical density and stabbed onto TY agar plates containing $20 \mathrm{mM} \mathrm{Glc}$, GlcN, or GlcNAc. The plates were incubated overnight, overlaid with soft agar containing S. sanguinis SK150, and then incubated for another $24 \mathrm{~h}$. The experiments were carried out in both aerobic and anaerobic environments.

communities in the oral cavity, we employed an established ex vivo model system (41, 42) in which cell-containing whole saliva (CCS) samples were pooled from 4 donors and used to seed biofilms on glass surfaces. A genetically marked S. mutans strain (UA159$\mathrm{Km}$ ) was spiked into the biofilms, and the effects of various sugars on persistence of $S$. mutans were monitored by CFU enumeration.

A schematic of the experimental design and various groups is shown in Fig. 7A. A biofilm medium (43) was used, but the carbohydrate content was modified such that Glc, GlcN, or GlcNAc was present at $18 \mathrm{mM}$ and $2 \mathrm{mM}$ sucrose was present in all 
A.

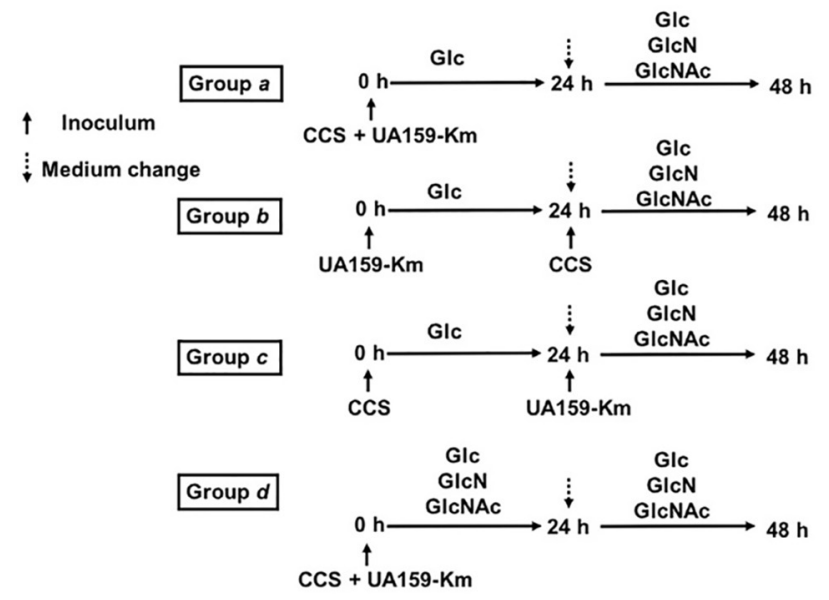

C.

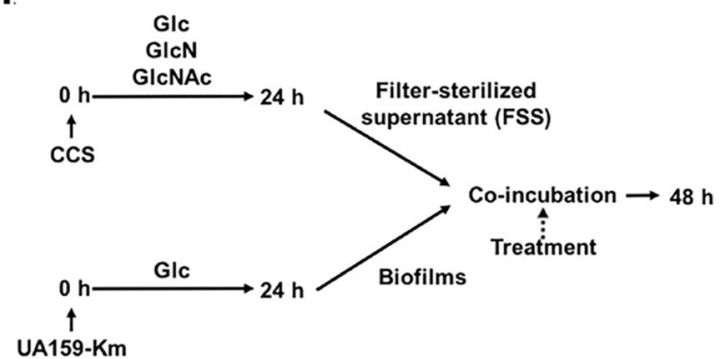

B.

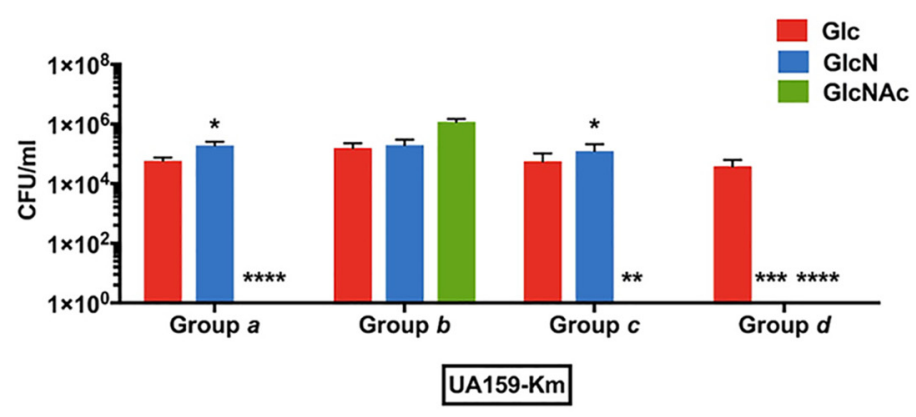

D.

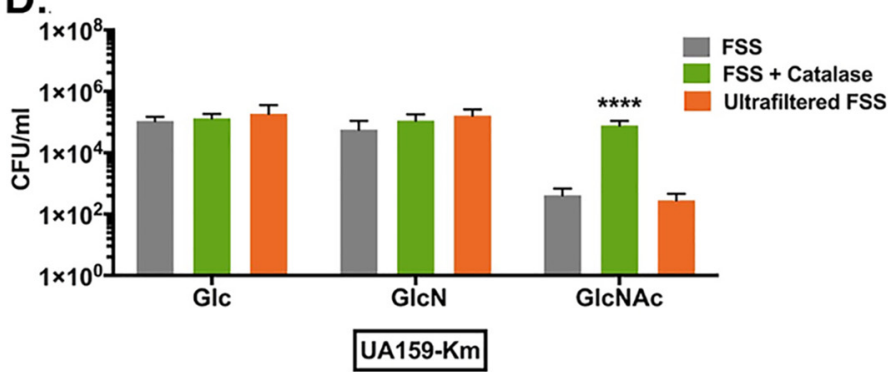

FIG 7 Amino sugars impact the survival of S. mutans in CCS-derived biofilms. (A and B) To initiate a biofilm, UA159-Km and cell-containing saliva (CCS) were used simultaneously (groups a and d) or $24 \mathrm{~h}$ apart (groups b and c) to inoculate BM supplemented with 2 mM sucrose and 18 mM various other carbohydrates. After $24 \mathrm{~h}$ of incubation, the biofilms were washed and resupplied with fresh medium and additional bacteria as specified (see Materials and Methods for details). (B) At the end of 2-day incubations, biofilms were processed to quantify the number of CFU of UA159-Km. Asterisks indicate statistically significant differences compared to cultures prepared with Glc. ${ }^{*}, P<0.05 ;{ }^{* *}, P<0.01 ;{ }^{* * *}, P<0.001 ;{ }^{* * *}, P<0.0001$. (C and D) A UA159-Km biofilm was treated for $24 \mathrm{~h}$ with a filter-sterilized supernate (FSS) derived from biofilm cultures of CCS that were formed aerobically under different carbohydrate conditions, followed by CFU quantification of UA159-Km (D). The FSS was treated with catalase, passed through an ultrafiltration device (MWCO, $10 \mathrm{kDa})$, or left untreated. Asterisks indicate statistically significant differences compared to untreated FSS. ${ }^{*}, P<0.05 ;{ }^{* *}, P<0.01 ;{ }^{* * *}, P<0.001 ;{ }^{* * * *}, P<0.0001$.

samples. In group a, S. mutans was coinoculated with CCS in BMGS (Glc) for the first $24 \mathrm{~h}$, and then the medium was replaced with BMGICNS (BM supplemented with $18 \mathrm{mM}$ GIcN and $2 \mathrm{mM}$ sucrose), BMGlcNAcS (BM supplemented with $18 \mathrm{mM}$ GlcNAc and $2 \mathrm{mM}$ sucrose), or BMGS for the next $24 \mathrm{~h}$ before plating. Compared with biofilms grown in BMGS only, the CFU of $S$. mutans in biofilms provided with GICN was 3.6-fold greater; however, CFU of $S$. mutans from GlcNAc-cultured biofilms were hardly detectable (Fig. 7B).

To determine whether the CCS inocula or S. mutans was able to first establish a biofilm, prior to inoculation with S. mutans or CCS, respectively, S. mutans (group b) or CCS (group c) was inoculated alone as the initial colonizer. CCS (b) or S. mutans (c) then was introduced on the following day; carbohydrate sources were varied as for group a. When the initial colonizer was S. mutans, slightly higher numbers of CFU of S. mutans were recovered from cultures grown with GlcNAc than with Glc or GICN (Fig. 7B, group b). In contrast, when the initial inoculum was CCS (group c), similar to what was seen in group a, very few S. mutans cells were detected in GICNAc biofilms, whereas the CFU of S. mutans recovered from GlcN biofilms were comparable to those of Glc-cultured biofilms.

We then examined how the biofilms would mature if the amino sugars were present from the time of initial inoculation (group d). In this case, CCS and S. mutans were coinoculated as group a and the cells were cultured in BM constituted with sucrose and either Glc, GlcN, or GlcNAc for 48 h. The resultant cell counts of S. mutans were below 
the limit of detection in GlcNAc-grown biofilms. Of note, unlike the three other experiments, treatment with GIcN for $48 \mathrm{~h}$ also drastically reduced the number of CFU of S. mutans recovered from the biofilms (Fig. 7B). Clearly, for this particular biofilm model, addition of GICNAc, and GICN for longer periods of time, could result in profound changes in the ecology of the microbiome, significantly affecting the persistence of $S$. mutans.

To determine if $\mathrm{H}_{2} \mathrm{O}_{2}$ or certain secreted factors were responsible for the diminished persistence of $S$. mutans associated with growth on amino sugars, UA159-Km alone was used to form biofilms on glass by incubating in BMGS for $24 \mathrm{~h}$. Twenty-four-h CCS biofilms were similarly prepared using BMGS, BMGIcNS, or BMGICNAcS (Fig. 7C). The supernatant fluids of each CCS biofilm culture were harvested and filter sterilized, and then a portion of the sample (filter-sterilized supernates [FSS]) was treated with catalase to degrade $\mathrm{H}_{2} \mathrm{O}_{2}$ (FSS plus catalase) and another aliquot was passed through an ultrafiltration device to eliminate constituents with a molecular mass of $>10 \mathrm{kDa}$ (ultrafiltered FSS). Along with untreated control FSS, these different FSS samples were used to treat 1-day S. mutans biofilms for $24 \mathrm{~h}$, followed by CFU enumeration of $S$. mutans. As shown in Fig. 7D, when FSS derived from Glc or GlcN cultures was added to the $S$. mutans biofilms, neither catalase treatment nor ultrafiltration had any notable impact on the survival of S. mutans. In stark contrast, for FSS prepared using GIcNAcbased cultures, catalase treatment significantly reduced the efficiency of FSS to inhibit the survival of S. mutans, whereas ultrafiltration had no effect.

Combining all the experimental settings, GlcNAc has been most effective in inhibiting S. mutans in this complex biofilm. Indeed, the largest amounts of $\mathrm{H}_{2} \mathrm{O}_{2}$ were measured in FSS from GICNAC-based CCS biofilms, approximately 6 times that from GlcN-based biofilms (Fig. S4), reaffirming the role of $\mathrm{H}_{2} \mathrm{O}_{2}$ in amino sugar-dependent impacts on antagonism of $S$. mutans by oral commensals. In a control experiment, FSS derived from anaerobically formed CCS biofilms were used to treat the same 1-day $S$. mutans biofilm for $24 \mathrm{~h}$. The resultant CFU counts of S. mutans showed no difference among three different carbohydrates (Fig. S5).

\section{DISCUSSION}

The concept that a variety of abundant commensal bacteria, particularly oral streptococci, have overtly beneficial properties that include the ability to moderate biofilm acidification and to antagonize the growth of caries pathogens is central to the current understanding of the ecological underpinnings of dental caries. Antimicrobial compounds and fitness-promoting activities of the pathogen(s) and commensals are key components of the interactions that drive biofilm formation, foster community stability, and lead to ecological changes during disease development. While dietary carbohydrates can drive the formation of caries, most of the time the oral microbiome relies on host and bacterially derived nutrient sources, with amino sugars being an abundant and bioenergetically favorable source of carbon, nitrogen, and energy. The goal of the present study was to assess the effects of two amino sugars, GlcN and GlcNAc, on some of the most abundant oral streptococcal species in the context of their overall fitness and antagonistic interactions with S. mutans. To that end, low-passagenumber isolates from five representative abundant oral Streptococcus species were selected from recently obtained strains (28) rather than using standard laboratory strains that had been extensively passaged in vitro. The five isolates selected also displayed a spectrum of activities in phenotypes that have been deemed critical to antagonism and $\mathrm{pH}$ moderation. Using a number of established model systems, this study demonstrated the profound ability of amino sugars to influence interbacterial interactions that are central for shaping the ecological balance and virulence potential of dental biofilms. Specifically, amino sugars enhanced $\mathrm{H}_{2} \mathrm{O}_{2}$ production by peroxidogenic streptococci in liquid cultures and on agar plates (S. oralis, S. cristatus, and $S$. gordonii). Moreover, commensal oral streptococci are generally considered less acid tolerant than S. mutans, and growth on GICN also resulted in significantly increased AD activity and culture $\mathrm{pH}$ in some of the commensals. Although not at all detrimental to 
S. mutans, production of ammonia via the $A D$ system and from amino sugars can protect the organisms against acid stress. Thus, the combination of arginine and amino sugars may be quite beneficial to commensals in competition with S. mutans and other caries pathogens, which gain their advantage over the commensals by virtue of their ability to grow and metabolize at lower $\mathrm{pH}$ values than the commensals. Consistent with these trends, amino sugars largely favored the dominance and survival of commensals and diminished the persistence of $S$. mutans in dual-species and ex vivo consortium biofilm models.

A significant finding arising from this study is the distinct effects exerted by GlcN and GICNAc on the selected commensals and the significant differences in behaviors and outcomes noted among the models, which are designed to examine certain behaviors, albeit in different contexts. For example, addition of GlcN, as opposed to GlcNAc, enhanced AD activity by most clinical commensals in liquid culture (Fig. 5). Furthermore, GlcN was notably more effective at stimulating anti-S. mutans activities on plates by $S$. cristatus and $S$. oralis under aerobic conditions, apparently due to $\mathrm{H}_{2} \mathrm{O}_{2}$ production. The presence of either amino sugar in liquid cultures resulted in comparable enhancements in $\mathrm{H}_{2} \mathrm{O}_{2}$ production by almost all the clinical commensals. Although GlcN promoted the secretion of mutacins by S. mutans to a greater extent than did GlcNAc (Fig. 6), overall these results provide support for the idea that GlcN is the better amino sugar for enhancing the competitiveness of the chosen commensals.

However, in the saliva-derived ex vivo biofilm model, where a far more complex community exists to compete against $S$. mutans, the biofilm as a whole responded favorably to GlcNAc against colonization by the pathogen, whereas GICN appeared to benefit commensals only if present for longer periods of time. Thus, it is apparent that the two amino sugars of interest can exert different impacts on the behaviors of both the commensals and S. mutans. It must be stressed that these in vitro models differ from one another in significant ways: they differ in oxygen tension, nutrient availability, colonization sequence, and biodiversity. In particular, saliva is a natural reservoir of indigenous bacteria found in the oral cavity. Not only does saliva harbor a microbial community that closely matches that of dental plaque in a given individual (44), it also contains factors that strongly influence the development and maintenance of the dental microbiome, including mucins, trace elements, and host- and microbiotaderived enzymes (38). Future studies will focus on exploring the mechanisms responsible for the distinct effects of amino sugars in the context of oral biofilm complexity and host factors.

While studying a complex biological system such as the dental biofilm, a reductive approach comes with benefits and potential deficiencies. Genomic heterogeneity and functional overlap in the microbiota have proven widespread among the numerous streptococcal species colonizing the oral cavity (45). It is generally accepted that exchange of genetic material is a common occurrence among many members of the oral microbiome, especially among streptococci. In this study, we have noted that the low-passage-number isolate $S$. sanguinis $\mathrm{BCCO} 4$ was partly responsive to amino sugars (GlcN only) in the dual-species biofilm model (Fig. 2) and inhibited mutacin production (Fig. 6) in a manner that is independent of oxygen. In particular, BCC04 showed no enhancement in its antagonism against $S$. mutans in the presence of amino sugars on plates (Fig. 4) and in the biofilms supported by GlcNAc, despite the fact that it produced significantly more $\mathrm{H}_{2} \mathrm{O}_{2}$ when supplied with either amino sugar. As a complement to these experiments, a commonly used laboratory strain, S. sanguinis SK150, was tested in the same dual-species biofilm model. The results showed a differential response to carbohydrates, with both amino sugars yielding significantly lower viable counts of $S$. mutans (see Fig. S6 in the supplemental material). These disparate phenotypes of $S$. sanguinis isolates are likely a result of intraspecies genomic heterogeneity that affects their competition with S. mutans. In fact, strain BCC04 produces robust biofilms in the presence of sucrose (Fig. S1), whereas SK150 does not (data not shown). Recently, it was reported that another laboratory strain of S. sanguinis, SK36, showed a lack of response to carbohydrate availability in its ability to produce $\mathrm{H}_{2} \mathrm{O}_{2}(46)$, echoing a previous 
finding that $s p x B$ expression in S. sanguinis was unresponsive to carbohydrate source (47). Comparative and functional genomic analyses currently being performed on these and other recent isolates of $S$. sanguinis could shed more light on the cause of these distinct phenotypes. In a separate study that focused on the effects of arginine on the antagonistic interactions between oral commensals and S. mutans, it was shown that enormous diversity exists among commensal streptococci, both in their antagonistic capabilities and their susceptibility to $S$. mutans (48). Clearly, probing the basis for phenotypic variation among commensals and pathogens (49) remains an essential goal in efforts to improve caries risk assessment and intervention.

Another notable finding from this study is the superior capacity of S. cristatus BCC13 and S. oralis BCCO2 to antagonize S. mutans, and both strains showed improved abilities to inhibit S. mutans when amino sugars were present (Fig. 2 and 4). While limited research has been conducted on the interactions between S. cristatus and S. mutans, it is established that $S$. cristatus is able to adversely affect the periodontal pathogen Porphyromonas gingivalis, both by repressing the expression of the primary fimbrial adhesin of $P$. gingivalis (50) and through production of $\mathrm{H}_{2} \mathrm{O}_{2}$ (51). As shown here, $S$. cristatus is clearly capable of disrupting the persistence of $S$. mutans by producing large amounts of $\mathrm{H}_{2} \mathrm{O}_{2}$ and potentially reducing the production of mutacins. Some recent reports have indicated that $S$. cristatus could be associated with severe early childhood caries; however, more research is needed to corroborate these findings $(52,53)$. Similarly, S. oralis BCC02 appeared highly inhibitory to S. mutans in biofilms, at least partly due to being an efficient $\mathrm{H}_{2} \mathrm{O}_{2}$ producer. $S$. oralis has been reported to maintain homeostasis in oral biofilms, primarily by suppressing S. mutans overgrowth (54) and influencing biofilm formation by S. mutans (43). Earlier studies also demonstrated the remarkable ability of $S$. oralis to produce hydrogen peroxide (55), which is corroborated by the present study.

In conclusion, this study demonstrated the capacity of amino sugars in promoting endogenous commensal bacteria to antagonize a major dental caries pathogen, $\mathrm{S}$. mutans. It is also clear that the effects of amino sugars on streptococci, both the pathogen and commensals alike, are quite complex. For example, while $S$. mutans alone has been shown to respond favorably to the presence of amino sugars by enhancing aciduricity (21) or the production of mutacins, cocultivation with the commensal species under the same conditions has resulted in greater reductions in persistence of the pathogen compared to that with glucose. Aside from the enhanced production of $\mathrm{H}_{2} \mathrm{O}_{2}$ by the commensals, another possible explanation for this outcome is that commensals actively subvert the competitiveness of $S$. mutans when amino sugars are present in sufficient concentrations (14). While we expand our efforts to identify amino sugar-responsive, antagonistic commensals, the availability of genomic information on these isolates (28) will greatly aid our understanding of the basis for phenotypic behaviors affected by amino sugars. Based on this and other studies, amino sugars may have benefits when included in prebiotic or synbiotic strategies for caries management.

\section{MATERIALS AND METHODS}

Bacterial strains and culture conditions. The bacterial strains used in this study are listed in Table 1. A series of clinical isolates belonging to different Streptococcus species were selected from caries-free plaque samples and characterized by $16 \mathrm{~S}$ rRNA gene sequencing. Five such isolates, S. sanguinis BCC04, S. gordonii BCC09, Streptococcus cristatus BCC13, Streptococcus oralis BCC02, and Streptococcus intermedius $\mathrm{BCC}$ 1, were also screened for their abilities to express the arginine deiminase (AD) enzyme and to antagonize S. mutans. All streptococcal strains, including S. mutans UA159 and a mutant derivative, UA159-Km, with a kanamycin resistance cassette that replaced the nonessential sucrose phosphorylase ( $g t f A$ ) gene by using plasmid pBGK2 (56), were maintained on brain heart infusion (BHI) (Difco Laboratories, Detroit, MI) agar plates. Liquid BHI was used to prepare starter cultures for assays. TY (3\% tryptone, $0.5 \%$ yeast extract) medium was used to grow cells for measurements of $\mathrm{H}_{2} \mathrm{O}_{2}$ production and AD activity and as the base for agar plates used in a competition assay and a bacteriocin production assay. TY medium, the chemically defined medium FMC (21), and a biofilm medium (BM) (43) were each formulated to contain various carbohydrates at specified concentrations. Bacterial planktonic or biofilm cultures in most experiments were incubated at $37^{\circ} \mathrm{C}$ in a $5 \% \mathrm{CO}_{2}$ aerobic environment or in anaerobic jars (BD, Franklin Lakes, NJ) as noted. 
Planktonic growth assays. Commensals were cultured overnight in $\mathrm{BHI}$ and subcultured in $\mathrm{BHI}$ broth to mid-exponential phase (optical density at $600 \mathrm{~nm}\left[\mathrm{OD}_{600}\right]$ of $\approx 0.5$ ) and then diluted 1:50 into modified FMC medium formulated with final concentrations of $10 \mathrm{mM}$ glucose (Glc), glucosamine (GlcN), or $\mathrm{N}$-acetyl-D-glucosamine (GlcNAc) (Sigma Millipore, St. Louis, MO). Cultures were maintained at $37^{\circ} \mathrm{C}$, and the $\mathrm{OD}_{600}$ was measured and recorded every 30 min using a Bioscreen $\mathrm{C}$ Lab system (Helsinki, Finland) for $24 \mathrm{~h}$. To reduce exposure to air, approximately $60 \mu \mathrm{l}$ of mineral oil was overlaid on each sample (57).

Biofilm assays. Overnight bacterial cultures were diluted 1:20 into fresh $\mathrm{BHI}$ medium and subcultured to an $\mathrm{OD}_{600}$ of $\approx 0.5$. Each culture of the commensal alone, or together with S. mutans UA159-Km in a 1:1 ratio, was prepared by diluting (1:100) into a final volume of $400 \mu \mathrm{l}$ of BM (43) that contained $18 \mathrm{mM}$ glucose (Glc) and $2 \mathrm{mM}$ sucrose (S). The cultures were kept in an Ibidi glass plate (Ibidi USA, Inc., Madison, WI). After $24 \mathrm{~h}$ of incubation under aerobic conditions, the spent medium was removed, the biofilms formed on the glass were gently washed twice with sterile BM, and $400 \mu \mathrm{l}$ of BM containing $20 \mathrm{mM} \mathrm{Glc}$, GlcN, or GlcNAc was added back to the wells. After another $24 \mathrm{~h}$ of incubation, the spent medium was removed and the biofilms were washed three times with sterile phosphate-buffered saline (PBS).

The washed biofilms were treated with a LIVE/DEAD BacLight bacterial viability reagent (Thermo Fisher Scientific, Waltham, MA), such that live bacteria produced green fluorescence (SYTO 9) and dead cells emitted red fluorescence (propidium iodide [PI]). The architecture of the biofilms was examined using a confocal laser scanning microscope (Leica, Wetzlar, Germany) equipped with a $60 \times$ oil immersion lens objective. VoxCell software (VisiTech International, Sunderland, United Kingdom) was used to acquire and process biofilm images. For SYTO 9, the excitation wavelength was set at $488 \mathrm{~nm}$ and emission wavelength at $525 \pm 25 \mathrm{~nm}$; for PI, excitation was set at $642 \mathrm{~nm}$ and emission observed at $695 \pm 53 \mathrm{~nm}$. Biofilm stacks were also rendered in three dimensions using Imaris software (Bitplane, Belfast, United Kingdom).

To quantify bacterial viability in a dual-species biofilm, the biofilm was scraped off the coverslips, resuspended in sterile PBS, and dispersed by sonication (FB120; Fisher Scientific) (30 s twice, 65\% power). After serial dilution, bacterial suspensions were spread onto BHI agar plates with or without $1 \mathrm{mg} \mathrm{ml}-1$ kanamycin (Sigma) to enumerate $S$. mutans and total bacteria, respectively.

Measurement of $\mathrm{H}_{2} \mathrm{O}_{2}$ and $A D$ activities. Levels of $\mathrm{H}_{2} \mathrm{O}_{2}$ in liquid TY cultures were measured by following an established protocol detailed elsewhere (14), and AD activity in bacterial cells was determined as previously described (58).

Plate-based competition assays. Overnight bacterial cultures in BHI were washed with sterile PBS and resuspended in fresh TY medium containing $20 \mathrm{mM}$ the specified carbohydrate, with the $\mathrm{OD}_{600}$ being adjusted to 0.5 . The cell suspensions $(6 \mu \mathrm{l})$ of $\mathrm{S}$. mutans UA159 and each commensal were spotted in close proximity on TY agar plates formulated with $20 \mathrm{mM} \mathrm{Glc}$, GlcN, or GlcNAc. Plates were incubated under aerobic or anaerobic conditions for $24 \mathrm{~h}$ at $37^{\circ} \mathrm{C}$ before being photographed.

Bacteriocin production assays. Secretion of bacteriocins by S. mutans on agar plates was assessed according to a protocol detailed elsewhere (14), with minor modifications. Briefly, overnight cultures of S. mutans UA159 and commensal strains grown in BHI were washed with sterile PBS and resuspended to an $\mathrm{OD}_{600}$ of 0.5 using fresh TY base medium supplemented with the desired carbohydrate. S. mutans UA159 alone or together with one commensal strain (at a ratio of 1:1) was inoculated by stabbing onto TY agar plates containing $20 \mathrm{mM} \mathrm{Glc}$, GlcN, or GlcNAc. After $24 \mathrm{~h}$ of incubation at $37^{\circ} \mathrm{C}$, each plate was overlaid with $8 \mathrm{ml}$ of warm soft agar ( $0.75 \%$ TY agar, prepared with the same sugar as that in the plate) containing $10^{7}$ cells of $S$. sanguinis SK150 as an indicator strain. Plates were incubated for an additional $24 \mathrm{~h}$ before examination for zones of inhibition of growth of S. sanguinis.

Microcosm ex vivo biofilm model. Cell-containing saliva (CCS) samples (59) were collected and prepared from four healthy adult volunteers, who were nonsmokers and had not taken antibiotics for at least 3 months (IRB201500497 at University of Florida). Equal volumes of untreated whole saliva from each volunteer were then pooled, glycerol was added to a final concentration of $25 \%$, and the CCS was stored in aliquots at $-80^{\circ} \mathrm{C}$. The pooled CCS was used as an inoculum for biofilm development by thawing of an aliquot and diluting 1:50 into BM. When needed, exponential-phase cultures of S. mutans UA159-Km were prepared in BHI broth and inoculated at a ratio of 1:1,000. For the biofilm experiments, BM was formulated with $2 \mathrm{mM}$ sucrose and $18 \mathrm{mM}$ glucose (BMGS), GIcN (BMGlcNS), or GlcNAc (BMGICNACS). Biofilms were allowed to form in Ibidi glass plates (Ibidi USA, Inc., Madison, WI) at $37^{\circ} \mathrm{C}$ in an aerobic atmosphere supplemented with $5 \% \mathrm{CO}_{2}$.

Four different biofilm treatments were employed, as detailed in Fig. 7A. Mixed cultures of S. mutans UA159-Km and CCS (group a), UA159-Km alone (group b), or CCS alone (group c) were inoculated into BMGS and incubated for $24 \mathrm{~h}$. After washing with sterile, sugar-free BM medium, cultures were resupplied with BMGS, BMGICNS, or BMGICNAcS, followed by $24 \mathrm{~h}$ of incubation. For group d, mixed cultures of UA159-Km and CCS were inoculated into BMGS, BMGICNS, or BMGICNACS. After $24 \mathrm{~h}$ of incubation, the supernates were replaced with the same, fresh medium and the biofilms were incubated for another $24 \mathrm{~h}$ before harvesting.

To study the nature of the antagonistic effectors in these biofilms, CCS was used as the inoculum for biofilm development in BMGS, BMGICNS, or BMGICNACS for $24 \mathrm{~h}$. At the same time, panels of 24-h UA159-Km biofilms were established in Ibidi plates using BMGS, as described above. Filter-sterilized supernates (FSS; filter diameter, $0.22 \mu \mathrm{m}$ ) were each prepared from the spent medium of CCS biofilms and divided into three equal parts: (i) FSS supplemented with $1 \%$ catalase, (ii) FSS passed through an Amicon filter with a molecular weight cutoff (MWCO) of $10 \mathrm{kDa}$, and (iii) untreated FSS. UA159-Km 
biofilms were washed twice with PBS, and then the various FSS samples were added and the biofilms were incubated for $24 \mathrm{~h}$ before harvesting.

To quantify the persistence of UA159-Km after these treatments, the biofilm cultures were washed twice with PBS, mechanically removed by scraping, and resuspended in $400 \mu \mathrm{l}$ of PBS, followed by sonication (30 s twice) to disperse the cells. The bacterial suspensions were then serially diluted and plated on $\mathrm{BHI}$ agar plates containing kanamycin. All of the plates were incubated for $48 \mathrm{~h}$ before CFU were counted.

Statistical analysis. One-way analysis of variance was performed to evaluate the significance of the comparisons from various experiments. The level of significance was determined at a $P$ value of $<0.05$.

\section{SUPPLEMENTAL MATERIAL}

Supplemental material for this article may be found at https://doi.org/10.1128/AEM .00370-19.

SUPPLEMENTAL FILE 1, PDF file, $0.5 \mathrm{MB}$.

\section{ACKNOWLEDGMENTS}

This work was supported by DE12236 and DE25832 from the National Institute of Dental and Craniofacial Research. We thank Tanaz Farivar for technical support. L.C. was supported in part by a grant from the China Scholarship Council (CSC).

\section{REFERENCES}

1. He X, Shi W. 2009. Oral microbiology: past, present and future. Int J Oral Sci 1:47-58. https://doi.org/10.4248/ijos.09029.

2. Flemming HC, Wingender J, Szewzyk U, Steinberg P, Rice SA, Kjelleberg S. 2016. Biofilms: an emergent form of bacterial life. Nat Rev Microbiol 14:563-575. https://doi.org/10.1038/nrmicro.2016.94.

3. Kolstad RA. 1976. Strain typing of oral streptococci by the use of bacterial antagonism. J Dent Res 55:A154-A165.

4. Kreth J, Zhang Y, Herzberg MC. 2008. Streptococcal antagonism in oral biofilms: Streptococcus sanguinis and Streptococcus gordonii interference with Streptococcus mutans. J Bacteriol 190:4632-4640. https://doi .org/10.1128/JB.00276-08.

5. Kassebaum NJ, Bernabe E, Dahiya M, Bhandari B, Murray CJ, Marcenes W. 2015. Global burden of untreated caries: a systematic review and metaregression. J Dent Res 94:650-658. https://doi.org/10.1177/ 0022034515573272.

6. Bowen WH, Burne RA, Wu H, Koo H. 2018. Oral biofilms: pathogens, matrix, and polymicrobial interactions in microenvironments. Trends Microbiol 26:229-242. https://doi.org/10.1016/j.tim.2017.09.008.

7. Baker JL, Faustoferri RC, Quivey RG, Jr. 2017. Acid-adaptive mechanisms of Streptococcus mutans-the more we know, the more we don't. Mol Oral Microbiol 32:107-117. https://doi.org/10.1111/omi.12162.

8. Sturr MG, Marquis RE. 1992. Comparative acid tolerances and inhibitor sensitivities of isolated F-ATPases of oral lactic acid bacteria. Appl Environ Microbiol 58:2287-2291.

9. Liao S, Klein MI, Heim KP, Fan Y, Bitoun JP, Ahn SJ, Burne RA, Koo H, Brady LJ, Wen ZT. 2014. Streptococcus mutans extracellular DNA is upregulated during growth in biofilms, actively released via membrane vesicles, and influenced by components of the protein secretion machinery. J Bacteriol 196:2355-2366. https://doi.org/10.1128/JB.01493-14.

10. Hossain MS, Biswas I. 2011. Mutacins from Streptococcus mutans UA159 are active against multiple streptococcal species. Appl Environ Microbiol 77:2428-2434. https://doi.org/10.1128/AEM.02320-10.

11. Gronroos L, Saarela M, Matto J, Tanner-Salo U, Vuorela A, Alaluusua S. 1998. Mutacin production by Streptococcus mutans may promote transmission of bacteria from mother to child. Infect Immun 66:2595-2600.

12. Merritt J, Qi F. 2012. The mutacins of Streptococcus mutans: regulation and ecology. Mol Oral Microbiol 27:57-69. https://doi.org/10.1111/j .2041-1014.2011.00634.x.

13. Liu YL, Nascimento M, Burne RA. 2012. Progress toward understanding the contribution of alkali generation in dental biofilms to inhibition of dental caries. Int J Oral Sci 4:135-140. https://doi.org/10.1038/ijos.2012 .54.

14. Huang X, Palmer SR, Ahn SJ, Richards VP, Williams ML, Nascimento MM, Burne RA. 2016. A highly arginolytic Streptococcus species that potently antagonizes Streptococcus mutans. Appl Environ Microbiol 82: 2187-2201. https://doi.org/10.1128/AEM.03887-15.

15. Sheiham A, James WP. 2015. Diet and dental caries: the pivotal role of free sugars reemphasized. J Dent Res 94:1341-1347. https://doi.org/10 $.1177 / 0022034515590377$.

16. Pitts NB, Zero DT, Marsh PD, Ekstrand K, Weintraub JA, Ramos-Gomez F, Tagami J, Twetman S, Tsakos G, Ismail A. 2017. Dental caries. Nat Rev Dis Primers 3:17030. https://doi.org/10.1038/nrdp.2017.30.

17. Adler CJ, Dobney K, Weyrich LS, Kaidonis J, Walker AW, Haak W, Bradshaw CJA, Townsend G, Sołtysiak A, Alt KW, Parkhill J, Cooper A. 2013. Sequencing ancient calcified dental plaque shows changes in oral microbiota with dietary shifts of the Neolithic and Industrial revolutions. Nat Genet 45:450-455. https://doi.org/10.1038/ng.2536.

18. Cheng X, Redanz S, Cullin N, Zhou X, Xu X, Joshi V, Koley D, Merritt J, Kreth J. 2018. Plasticity of the pyruvate node modulates hydrogen peroxide production and acid tolerance in multiple oral streptococci. Appl Environ Microbiol 84:e01697-17. https://doi.org/10.1128/AEM .01697-17.

19. Zeng L, Dong Y, Burne RA. 2006. Characterization of cis-acting sites controlling arginine deiminase gene expression in Streptococcus gordonii. J Bacteriol 188:941-949. https://doi.org/10.1128/JB.188.3.941-949 .2006 .

20. Zeng L, Farivar T, Burne RA. 2016. Amino sugars enhance the competitiveness of beneficial commensals with Streptococcus mutans through multiple mechanisms. Appl Environ Microbiol 82:3671-3682. https://doi .org/10.1128/AEM.00637-16.

21. Moye ZD, Burne RA, Zeng L. 2014. Uptake and metabolism of $\mathrm{N}$-acetylglucosamine and glucosamine by Streptococcus mutans. Appl Environ Microbiol 80:5053-5067. https://doi.org/10.1128/AEM.00820-14.

22. Trent MS. 2004. Biosynthesis, transport, and modification of lipid A. Biochem Cell Biol 82:71-86. https://doi.org/10.1139/o03-070.

23. Schaffer C, Messner P. 2005. The structure of secondary cell wall polymers: how Gram-positive bacteria stick their cell walls together. Microbiology 151:643-651. https://doi.org/10.1099/mic.0.27749-0.

24. Battistone GC, Burnett GW. 1961. The free amino acid composition of human saliva. Arch Oral Biol 3:161-170. https://doi.org/10.1016/0003 -9969(61)90133-9.

25. Mandel ID, Hampar B, Thompson RH, Jr, Ellison SA. 1961. The carbohydrates of human parotid saliva. Arch Oral Biol 3:278-282.

26. Mandel ID, Thompson R, Jr, Ellison SA. 1964. The carbohydrate components of human submaxillary saliva. Arch Oral Biol 9:601-609. https:// doi.org/10.1016/0003-9969(64)90074-3.

27. Dobrogosz WJ. 1968. Effect of amino sugars on catabolite repression in Escherichia coli. J Bacteriol 95:578-584.

28. Velsko IM, Chakraborty B, Nascimento MM, Burne RA, Richards VP. 2018. Species designations belie phenotypic and genotypic heterogeneity in oral streptococci. mSystems 3:e00158-18. https://doi.org/10.1128/ mSystems.00158-18.

29. Zeng L, Martino NC, Burne RA. 2012. Two gene clusters coordinate galactose and lactose metabolism in Streptococcus gordonii. Appl Environ Microbiol 78:5597-5605. https://doi.org/10.1128/AEM.01393-12. 
30. Marquis RE. 1995. Oxygen metabolism, oxidative stress and acid-base physiology of dental plaque biofilms. J Ind Microbiol 15:198-207. https://doi.org/10.1007/BF01569826.

31. Zheng CY, Pan J, Wang L, Zhang CF. 2011. Effects of hydrogen peroxidecontaining bleaching on cariogenic bacteria and plaque accumulation. Chin J Dent Res 14:47-52.

32. Whiley RA, Fraser H, Hardie JM, Beighton D. 1990. Phenotypic differentiation of Streptococcus intermedius, Streptococcus constellatus, and Streptococcus anginosus strains within the "Streptococcus milleri group". J Clin Microbiol 28:1497-1501.

33. Zheng L, Itzek A, Chen Z, Kreth J. 2011. Environmental influences on competitive hydrogen peroxide production in Streptococcus gordonii. Appl Environ Microbiol 77:4318-4328. https://doi.org/10.1128/AEM .00309-11.

34. Ahn SJ, Wen ZT, Burne RA. 2007. Effects of oxygen on virulence traits of Streptococcus mutans. J Bacteriol 189:8519-8527. https://doi.org/10 .1128/JB.01180-07.

35. Dong Y, Chen YY, Burne RA. 2004. Control of expression of the arginine deiminase operon of Streptococcus gordonii by CcpA and Flp. J Bacteriol 186:2511-2514. https://doi.org/10.1128/JB.186.8.2511-2514.2004.

36. Balakrishnan M, Simmonds RS, Kilian M, Tagg JR. 2002. Different bacteriocin activities of Streptococcus mutans reflect distinct phylogenetic lineages. J Med Microbiol 51:941-948. https://doi.org/10.1099/0022 -1317-51-11-941.

37. Wang BY, Kuramitsu HK. 2005. Interactions between oral bacteria: inhibition of Streptococcus mutans bacteriocin production by Streptococcus gordonii. Appl Environ Microbiol 71:354-362. https://doi.org/10.1128/ AEM.71.1.354-362.2005.

38. Amerongen AV, Veerman EC. 2002. Saliva-the defender of the oral cavity. Oral Dis 8:12-22. https://doi.org/10.1034/j.1601-0825.2002.10816.x.

39. Foster JS, Kolenbrander PE. 2004. Development of a multispecies oral bacterial community in a saliva-conditioned flow cell. Appl Environ Microbiol 70:4340-4348. https://doi.org/10.1128/AEM.70.7.4340-4348 .2004 .

40. Ledder RG, Gilbert P, Pluen A, Sreenivasan PK, De Vizio W, McBain AJ. 2006. Individual microflora beget unique oral microcosms. J Appl Microbiol 100: 1123-1131. https://doi.org/10.1111/j.1365-2672.2006.02847.x.

41. Kolderman E, Bettampadi D, Samarian D, Dowd SE, Foxman B, Jakubovics NS, Rickard AH. 2015. L-Arginine destabilizes oral multi-species biofilm communities developed in human saliva. PLoS One 10:e0121835. https://doi.org/10.1371/journal.pone.0121835.

42. Fernandez CE, Aspiras MB, Dodds MW, Gonzalez-Cabezas C, Rickard AH. 2017. The effect of inoculum source and fluid shear force on the development of in vitro oral multispecies biofilms. J Appl Microbiol 122:796-808. https://doi.org/10.1111/jam.13376.

43. Wen ZT, Yates D, Ahn SJ, Burne RA. 2010. Biofilm formation and virulence expression by Streptococcus mutans are altered when grown in dual-species model. BMC Microbiol 10:111. https://doi.org/10.1186/1471 -2180-10-111.

44. Yamanaka W, Takeshita T, Shibata Y, Matsuo K, Eshima N, Yokoyama T, Yamashita Y. 2012. Compositional stability of a salivary bacterial population against supragingival microbiota shift following periodontal therapy. PLoS One 7:e42806. https://doi.org/10.1371/journal.pone.0042806.

45. Palmer SR, Miller JH, Abranches J, Zeng L, Lefebure T, Richards VP, Lemos JA, Stanhope MJ, Burne RA. 2013. Phenotypic heterogeneity of genomically-diverse isolates of Streptococcus mutans. PLoS One 8:e61358. https://doi.org/10.1371/journal.pone.0061358.
46. Redanz S, Masilamani R, Cullin N, Giacaman RA, Merritt J, Kreth J. 2018 Distinct regulatory role of carbon catabolite protein $A(C \mathrm{cpA})$ in oral streptococcal spxB expression. J Bacteriol 200:e00619-17. https://doi .org/10.1128/JB.00619-17.

47. Zheng L, Chen Z, Itzek A, Ashby M, Kreth J. 2011. Catabolite control protein $A$ controls hydrogen peroxide production and cell death in Streptococcus sanguinis. J Bacteriol 193:516-526. https://doi.org/10 .1128/JB.01131-10.

48. Huang $X$, Browngardt CM, Jiang M, Ahn SJ, Burne RA, Nascimento MM 2018. Diversity in antagonistic interactions between commensal oral streptococci and Streptococcus mutans. Caries Res 52:88-101. https:// doi.org/10.1159/000479091.

49. Liu Y, Palmer SR, Chang H, Combs AN, Burne RA, Koo H. 2018. Differential oxidative stress tolerance of Streptococcus mutans isolates affects competition in an ecological mixed-species biofilm model. Environ Microbiol Rep 10:12-22. https://doi.org/10.1111/1758-2229.12600.

50. Christopher AB, Arndt A, Cugini C, Davey ME. 2010. A streptococcal effector protein that inhibits Porphyromonas gingivalis biofilm development. Microbiology 156:3469-3477. https://doi.org/10.1099/mic.0.042671-0.

51. Herrero ER, Slomka V, Bernaerts K, Boon N, Hernandez-Sanabria E, Passoni BB, Quirynen M, Teughels W. 2016. Antimicrobial effects of commensal oral species are regulated by environmental factors. J Dent 47:23-33. https://doi.org/10.1016/j.jdent.2016.02.007.

52. Tanner AC, Mathney JM, Kent RL, Chalmers NI, Hughes CV, Loo CY, Pradhan N, Kanasi E, Hwang J, Dahlan MA, Papadopolou E, Dewhirst FE. 2011. Cultivable anaerobic microbiota of severe early childhood caries. J Clin Microbiol 49:1464-1474. https://doi.org/10.1128/JCM.02427-10.

53. Dzidic M, Collado MC, Abrahamsson T, Artacho A, Stensson M, Jenmalm MC, Mira A. 2018. Oral microbiome development during childhood: an ecological succession influenced by postnatal factors and associated with tooth decay. ISME J 12:2292-2306. https://doi.org/10.1038/s41396 -018-0204-z.

54. Thurnheer T, Belibasakis GN. 2018. Streptococcus oralis maintains homeostasis in oral biofilms by antagonizing the cariogenic pathogen Streptococcus mutans. Mol Oral Microbiol 33:234-239. https://doi.org/ 10.1111/omi.12216.

55. García-Mendoza A, Liébana J, Castillo AM, de la Higuera A, Piédrola G. 1993. Evaluation of the capacity of oral streptococci to produce hydrogen peroxide. J Med Microbiol 39:434-439. https://doi.org/10.1099/ 00222615-39-6-434.

56. Wen ZT, Burne RA. 2001. Construction of a new integration vector for use in Streptococcus mutans. Plasmid 45:31-36. https://doi.org/10.1006/ plas.2000.1498.

57. Zeng L, Burne RA. 2010. Seryl-phosphorylated HPr regulates CcpAindependent carbon catabolite repression in conjunction with PTS permeases in Streptococcus mutans. Mol Microbiol 75:1145-1158. https:// doi.org/10.1111/j.1365-2958.2009.07029.x.

58. Rickard AH, Gilbert P, High NJ, Kolenbrander PE, Handley PS. 2003. Bacterial coaggregation: an integral process in the development of multi-species biofilms. Trends Microbiol 11:94-100. https://doi.org/10 .1016/S0966-842X(02)00034-3.

59. Nance WC, Dowd SE, Samarian D, Chludzinski J, Delli J, Battista J, Rickard AH. 2013. A high-throughput microfluidic dental plaque biofilm system to visualize and quantify the effect of antimicrobials. J Antimicrob Chemother 68:2550-2560. https://doi.org/10.1093/jac/dkt211. 